\title{
Structural insight into substrate specificity of human intestinal maltase-glucoamylase
}

\author{
Limei Ren ${ }^{1,2^{*}}$, Xiaohong Qin ${ }^{1,3^{*}}$, Xiaofang Cao ${ }^{1,2}$, Lele Wang ${ }^{1,3}$, Fang Bai ${ }^{2}$, Gang Bai ${ }^{1,2} \bowtie$, Yuequan Shen ${ }^{1,3 凶}$ \\ ${ }^{1}$ State Key Laboratory of Medicinal Chemical Biology, Nankai University, Tianjin 300071, China \\ 2 College of Pharmacy, Nankai University, Tianjin 300071, China \\ ${ }^{3}$ College of Life Sciences, Nankai University, Tianjin 300071, China \\ $\triangle$ Correspondence: gangbai@nankai.edu.cn (G. Bai); yshen@nankai.edu.cn, yuequan74@yahoo.com (Y. Shen) \\ Received September 8, 2011 Accepted September 15, 2011
}

\begin{abstract}
Human maltase-glucoamylase (MGAM) hydrolyzes linear alpha-1,4-linked oligosaccharide substrates, playing a crucial role in the production of glucose in the human lumen and acting as an efficient drug target for type 2 diabetes and obesity. The amino- and carboxyl-terminal portions of MGAM (MGAM-N and MGAM-C) carry out the same catalytic reaction but have different substrate specificities. In this study, we report crystal structures of MGAM-C alone at a resolution of $3.1 \AA$, and in complex with its inhibitor acarbose at a resolution of $2.9 \AA$. Structural studies, combined with biochemical analysis, revealed that a segment of 21 amino acids in the active site of MGAM-C forms additional sugar subsites $(+2$ and +3 subsites), accounting for the preference for longer substrates of MAGM-C compared with that of MGAM-N. Moreover, we discovered that a single mutation of Trp1251 to tyrosine in MGAM-C imparts a novel catalytic ability to digest branched alpha-1,6-linked oligosaccharides. These results provide important information for understanding the substrate specificity of alphaglucosidases during the process of terminal starch digestion, and for designing more efficient drugs to control type 2 diabetes or obesity.
\end{abstract}

KEYWORDS MGAM C-terminal domain, inhibitor, crystal structure, acarbose, type 2 diabetes

\section{INTRODUCTION}

Starches are one of the primary energy sources for humans and other animals. To digest dietary starches in the human digestive system completely, four a-glucosidases are involved (Van Beers et al., 1995; Nichols et al., 2009). The initial digestion is carried out by salivary and pancreatic $\alpha$ amylase. This step produces maltose, maltotriose, and other $\alpha-1,6$ and $\alpha-1,4$ oligoglucans (Brayer et al., 2000). The resultant mixture is eventually hydrolyzed into glucose by maltase-glucoamylase (MGAM; EC 3.2.1.20 and 3.2.1.3) and sucrase-isomaltase (SI; EC 3.2.148 and 3.2.10) in the lumen (Dahlqvist and Telenius, 1969; Semenza, 1986). A strong synergism is proposed to exist between these four enzymes in order to allow for complete digestion (Quezada-Calvillo et al., 2008). Starch metabolic disorder is directly related to many human diseases, including type 2 diabetes, which is a major global health concern (Low, 2010). Extensive studies have focused on these a-glucosidases, and controlling their activities has proved efficient in controlling postprandial blood glucose levels with the aim of reducing the risk of complications in diabetic patients (Jenkins et al., 1981; Brayer et al., 1995; Rabasa-Lhoret and Chiasson, 1998; Qin et al., 2011).

Both MGAM and SI are enzymes with duplicated catalytic centers that anchor on the small-intestinal brush-border membrane via an O-glycosylated link stemming from their $\mathrm{N}$-termini (Sim et al., 2008). Both enzymes can be divided into an N-terminal subunit (MGAM-N and SI-N) and a C-terminal subunit (MGAM-C and SI-C) (Nichols et al., 1998; Nichols et al., 2003). According to the carbohydrate-active enzymes (CAZY) classification system, all of these subunits belong to the glycoside hydrolase 31 family (GH31) subgroup 1 , which contains the characteristic sequence WiDMNE in their catalytic centers (Ernst et al., 2006). The sequence identity between MGAM-N and IS-N is high, being close to $60 \%$, which is more than the percentage of identity between their respective C-terminal subunits in the same protein $(40 \%)$. All

"These authors contributed equally to the work. 
A

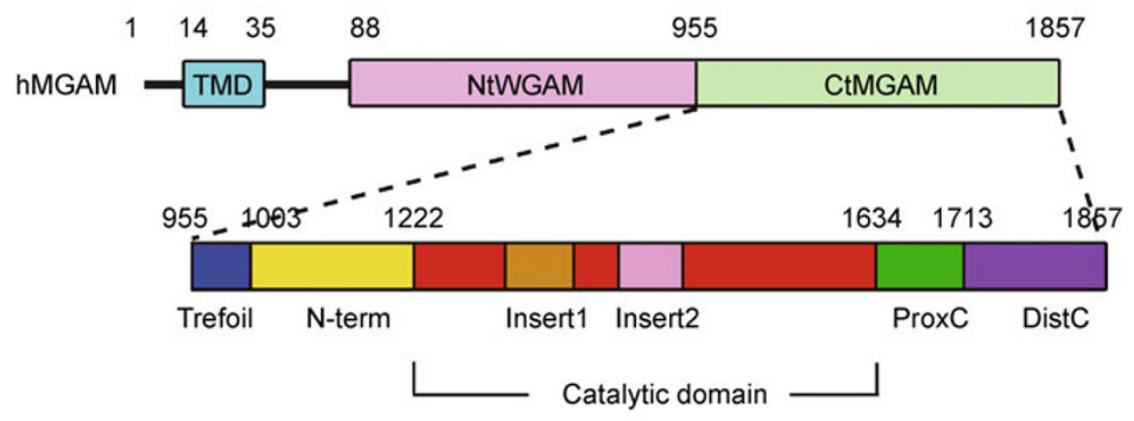

B

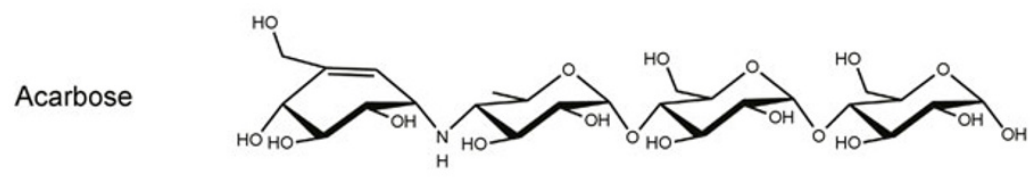

C

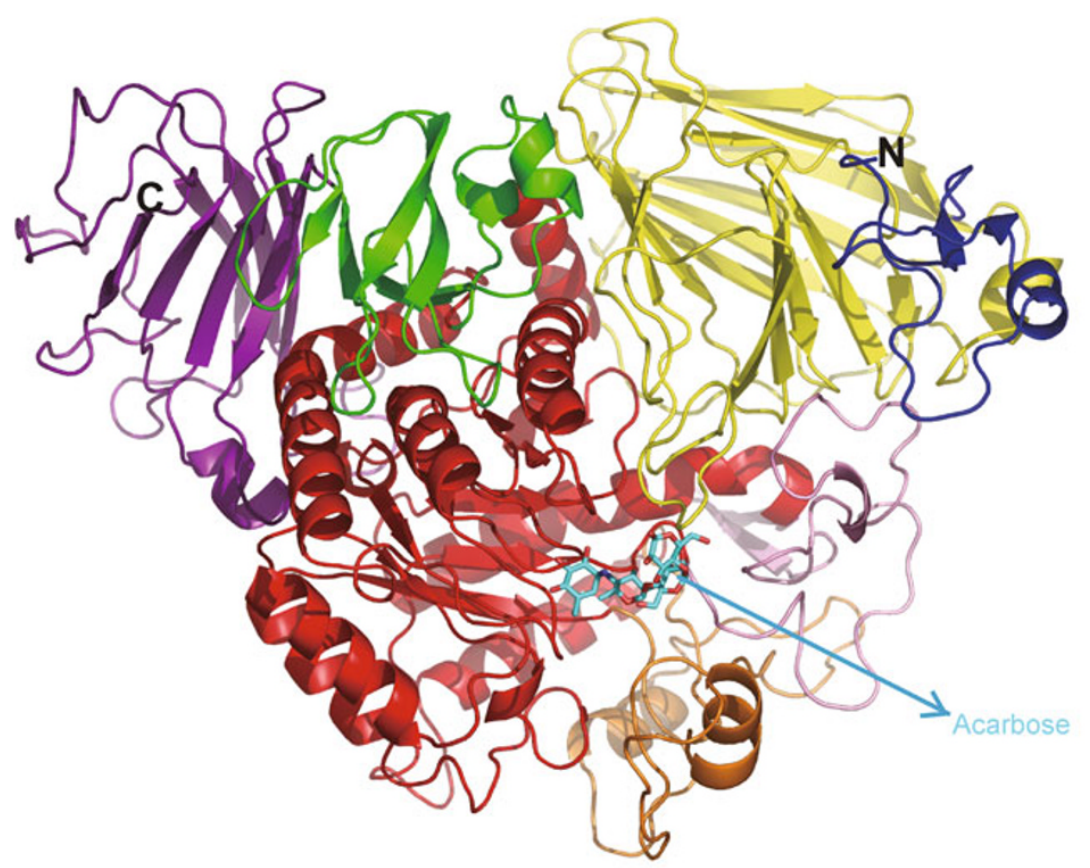

Figure 1. Structure of the MGAM-C/acarbose complex. (A) Schematic representation of human MGAM domains with aminoacid boundaries; (B) Schematic drawing of the structure of acarbose; (C) Ribbon diagram of the MGAM-C/acarbose complex. Individual domains are colored as follows: trefoil Type-P domain (blue), N-terminal domain (yellow), catalytic ( $\beta / \alpha) 8$ domain (red), catalytic domain Insert 1 (orange), catalytic domain Insert 2 (pink), proximal C-terminal domain (ProxC) (green), and distal Cterminal domain (DistC) (purple). The bound inhibitor acarbose is shown as a stick model and colored cyan. N, N terminal; C, C terminal.

four subunits exhibit similar exoglucosidase activities against linear $\alpha-1,4$-linked maltose substrates but different preferences for oligosaccharides substrates with various lengths (Sim et al., 2010). MGAM-N and SI-N both have maximal activity against substrates with two glucoses, while MGAM-C and SI-C prefer oligosaccharides with up to four glucose residues (Heymann et al., 1995; Quezada-Calvillo et al., 2008). Additionally, SI displays activities against $\alpha-1,2-$ and $\alpha-$ 1,6-linked oligoglucans (Nichols et al., 2009). Crystal structures of both MGAM-N and SI-N are known (Sim et al., 2008, 2010), and structural analyses show that they are highly similar in their overall folding patterns. However, little is known about the molecular mechanisms underlying the substrate length preferences of the $\mathrm{N}$ - and $\mathrm{C}$-terminal catalytic units.

MGAM-C has a molecular weight of $\sim 100 \mathrm{kDa}$ (Fig. $1 \mathrm{~A}$ and $1 B)$. MGAM-C has much higher activity than MGAM-N and is actually the subunit with the highest activity among these 
four "maltase" subunits (Quezada-Calvillo et al., 2008). Consequently, inhibition of the activity of MGAM-C has proven to be an efficient treatment for some diseases, such as type 2 diabetes or obesity (Rossi et al., 2006). Acarbose, an oral anti-diabetic medicine currently in use, shows a stronger level of inhibition against MGAM-C than MGAM-N (Sim et al., 2010). To investigate the structural basis for the substrate specificity of MGAM-C, we solved the crystal structures of MGAM-C alone and in complex with acarbose. Structural analyses combined with biochemical results revealed new subsites for substrate binding in this type of a-glucosidase and uncovered the molecular mechanism for the different substrate specificities of MGAM-C, MGAM-N and SI-N.

\section{RESULTS}

\section{Overall structure of MGAM-C in complex with acarbose}

MGAM-C proteins were produced in the Pichia pastoris yeast expression system and were purified to homogeneity for crystallization. Crystals of MGAM-C belong to the space group $P 4_{3}{ }_{1} 2$, with unit cell dimensions $a=b=106.23 \AA$, and $c=517.56 \AA$. Such a long $c$ axis is notably rare in protein crystals. The crystal structure of human MGAM-C in complex with acarbose (MGAM-C/acarbose) was finally determined at a resolution of $2.9 \AA$. The complex structure was solved by molecular replacement using the structure of MGAM-N (PDB code: $2 \mathrm{QLY}$ ) as a template. Each asymmetric unit contains two MGAM-C molecules. The two molecules in the asymmetric unit resemble each other, with a root-mean-square deviation (RMSD) value of $0.25 \AA$ for the $890 \mathrm{C} \alpha$ atoms. We will only refer to molecule $A$ in the following discussion.

The interface between the two molecules, with a surface area of $670 \AA^{2}$, as calculated by the program AREAIMOL (Lee and Richards, 1971), presumably indicates the presence of a crystallographic dimer, rather than a stable dimer in solution. The monomer state of MGAM-C in solution was also confirmed by analytical ultracentrifugation (Fig. S1). The structure of human MGAM-C alone was determined to a resolution of $3.1 \AA$ and solved by a difference Fourier method using as a final model for the complex structure of MGAM-C/ acarbose. A structural comparison between MGAM-C alone and in complex with acarbose did not show any major conformational changes.

The MGAM-C structure can be divided into five major domains: a trefoil Type-P domain (residues 955-1001); an Nterminal domain (residues 1002-1220) composed of a series of anti-parallel $\beta$-barrels; a catalytic domain (residues $1221-1632)$ consisting of a $(\beta / \alpha)_{8}$-barrel with two loop inserts (Insert 1, residues 1317-1386 and Insert 2, residues 1424-1477); a proximal C-terminal domain (residues 1633-1711) and a distal C-terminal domain (residues 1712-1857) (Fig. 1C). The last four residues in the distal Cterminal domain are disordered in both crystals and were not included in the final models.

The overall architectural fold of MGAM-C is similar to those of MGAM-N (PDB code 2QLY) and SI-N (PDB code 3LPP) (Fig. S2). The superposition of MGAM-C onto MGAM-N or SI$\mathrm{N}$ gives an RMSD value of $1.28 \AA$ for $815 \mathrm{C} \alpha$ atoms or $1.27 \AA$ for $822 \mathrm{C} \alpha$ atoms, respectively.

\section{Active site}

Acarbose, a pseudo-tetrasaccharide that is composed of an acarviosine group a-(1-4) linked to a maltose, is a competitive inhibitor of MGAM-C (Fig. S3). In our complex structure, acarbose was found in the active site of MGAM-C (Fig. 2A).

Acarbose spans subsites from -1 to +3 of MGAM-C, with its non-hydrolyzable $\mathrm{N}$-linked bond occupying the catalytic center. Numerous hydrogen bonds and hydrophobic interactions are involved in the interactions between MGAM-C and acarbose (Fig. 2A and 2B). At subsite -1 , atoms NE2 of His1584 and OD2 of Asp1279 form hydrogen bonds with chemical groups $\mathrm{C} 3-\mathrm{OH}$ and $\mathrm{C} 4-\mathrm{OH}$ of the unsaturated cyclitol unit of acarbose. Additional stabilization of the first sugar ring may result from hydrophobic interactions with bulk side chains of residues Tyr1251, Trp1523 and Trp1418. At subsite +1 , the side chains of Asp1157 form two hydrogen bonds with the $\mathrm{C} 2-\mathrm{OH}$ and $\mathrm{C} 3-\mathrm{OH}$ groups of 4,6-dideoxy-4amino- $D$-glucose of acarbose. Additionally, atom $\mathrm{NH} 1$ of Arg1510 makes a hydrogen bond with the $\mathrm{C} 3-\mathrm{OH}$ group of the second ring. Residues Trp1355 and Phe1559 stack with the first and second rings of acarbose, further stabilizing the acarbose molecule. Most importantly, the residue Asp1526 forms one hydrogen bond with atom N4B of acarbose, which is a candidate for an acid/base catalytic residue. At subsite +2 , the side chain of residue Trp1369 stacks with the third ring of acarbose. At subsite +3 , the two residues Phe1560 and Pro1159 stabilize the fourth ring through hydrophobic interactions.

\section{Substrate specificity}

Kinetic studies showed that MGAM-N has similar binding constants for substrates G2-G6 (the oligosaccharide substrates with 2-7 rings referred to as $\mathrm{G} 2-\mathrm{G} 7$ ), with $K_{\mathrm{m}}$ values of $\sim 6 \mathrm{mmol} / \mathrm{L}$ (Table 1), while MGAM-C has a clear preference for G3-G6 substrates ( 1 mmol/L) over the $\mathrm{G} 2$ substrate maltose $(5.67 \mathrm{mmol} / \mathrm{L})$, indicating that major differences exist in the active sites of MGAM-N and MGAM-C. We also found that MGAM-N and MGAM-C show different inhibitor tolerances. Acarbose with four rings displayed much higher inhibitory activity against MGAM-C (3.44 \pm $0.10 \mu \mathrm{mol} / \mathrm{L})$ than against MGAM-N $(62 \pm 13 \mu \mathrm{mol} / \mathrm{L})$, thereby suggesting that MGAM-C has a higher binding affinity for longer substrates than does MGAM-N. Furthermore, fulllength human MGAM (Heymann et al., 1995) showed a similar pattern of substrate preference for G3-G6 $\left(K_{\mathrm{m}}\right.$ $\sim 1 \mathrm{mmol} / \mathrm{L}$ ) with $\mathrm{G} 2$ (Table 1). 
A
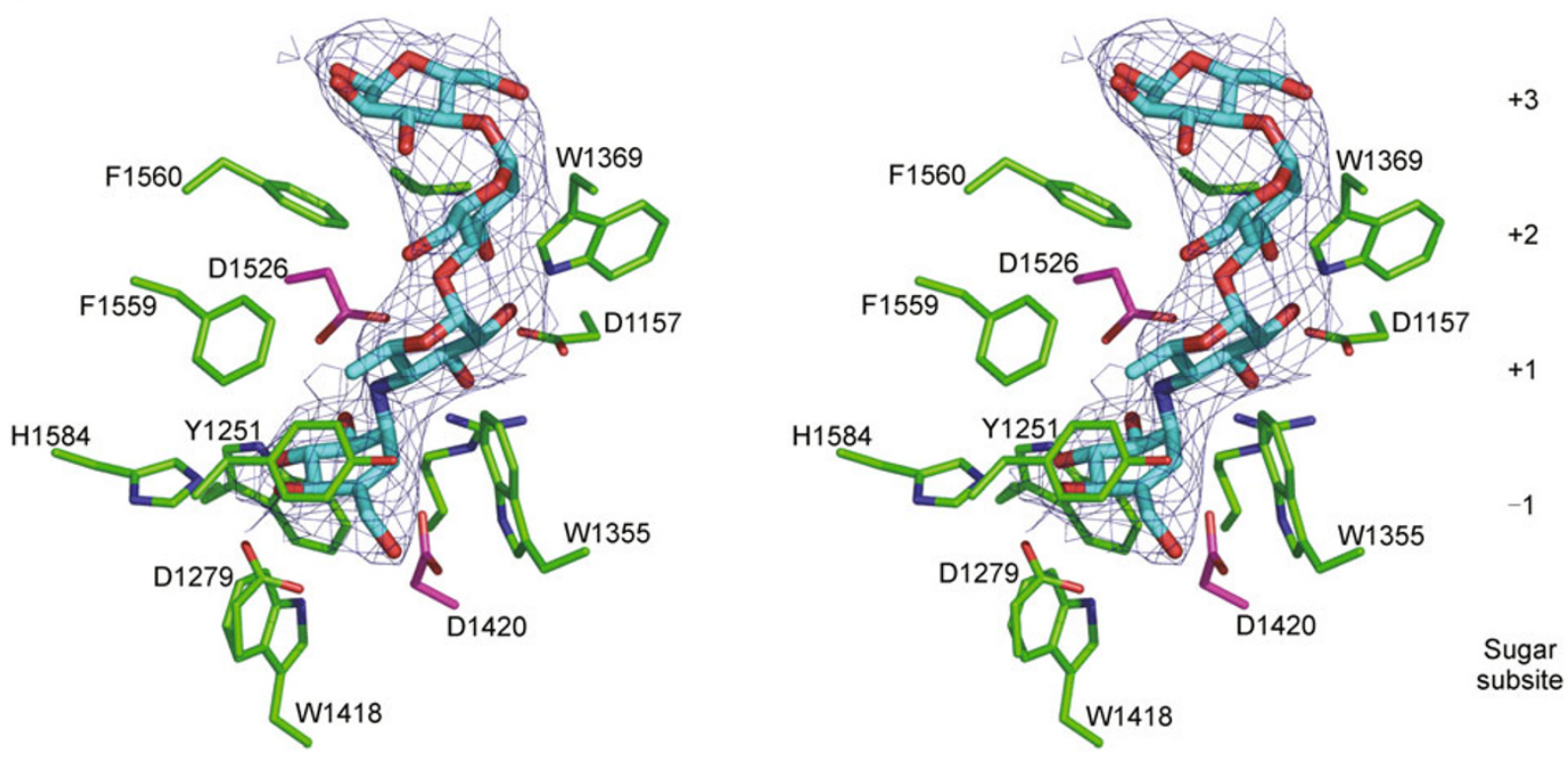

B

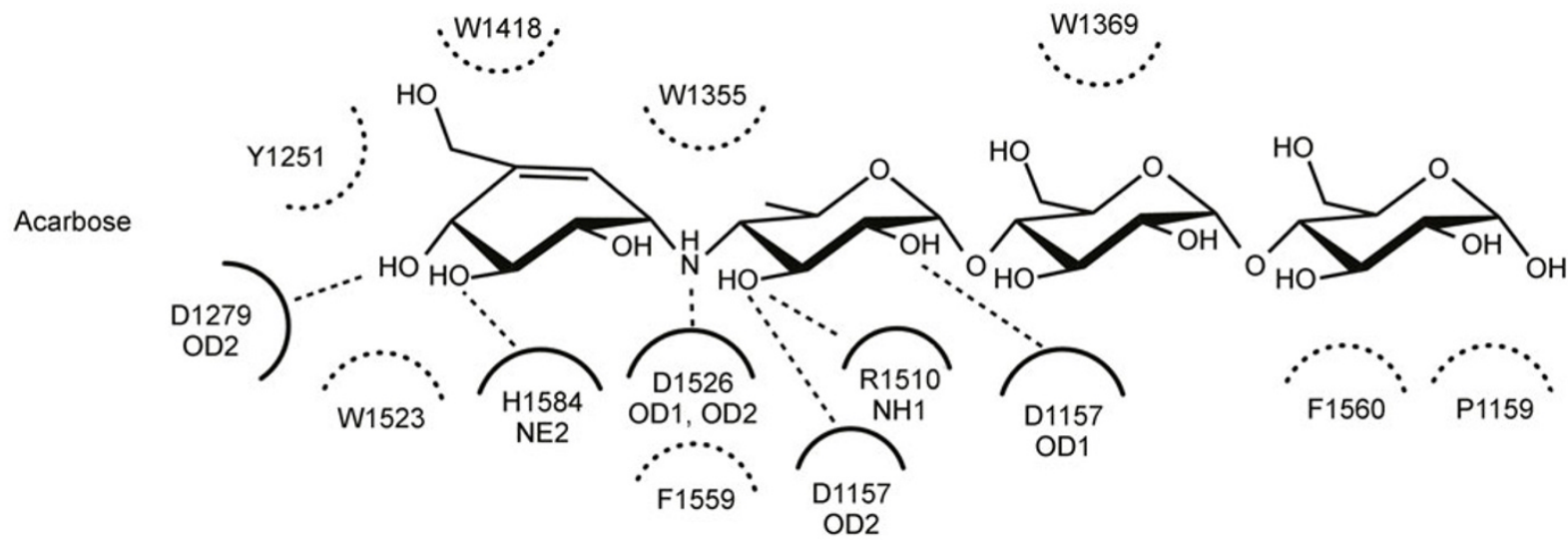

Sugar subsite
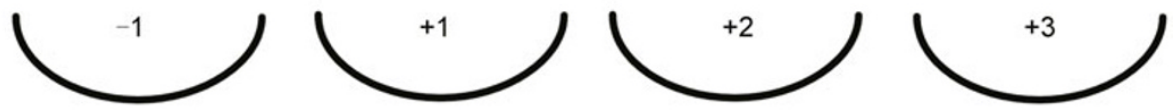

Figure 2. Interaction of MGAM-C with acarbose. (A) Stereo view of the 2Fo-Fc electron density map in the active site of the MGAM-C/acarbose structure contoured at the $2.0 \sigma$ level and shown in blue. Acarbose is represented as thick cyan sticks, and the active-site residues are represented as thin green sticks. (B) The diagrammatic representations of the hydrogen bonds (dashed lines) and hydrophobic interactions (dashed-lined semicircles) formed by MGAM-C with inhibitor acarbose. Sugar subsites $(-1,+1,+2$ and +3 ) are labeled accordingly.

Table 1 Kinetic parameters for wild-type and mutant MGAM

\begin{tabular}{lccc}
\hline Substrate & & $K_{\mathrm{m}}(\mathrm{mmol} / \mathrm{L})$ & MGAM-C-deltaS \\
\cline { 2 - 4 } & MGAM-N & MGAM-C & $5.91 \pm 0.72$ \\
Maltose & $6.40 \pm 0.58$ & $5.67 \pm 0.26$ & $1.89 \pm 0.37$ \\
Maltotriose & $4.44 \pm 0.57$ & $0.91 \pm 0.11$ & $4.73 \pm 0.36$ \\
Maltotetraose & $3.39 \pm 0.31$ & $0.96 \pm 0.06$ & $8.24 \pm 1.25$ \\
Maltopentaose & $9.14 \pm 0.24$ & $0.61 \pm 0.06$ & $12.33 \pm 0.91$ \\
Maltohexaose & $9.76 \pm 0.21$ & $1.05 \pm 0.10$ & $13.70 \pm 1.78$ \\
Maltoheptaose & $13.12 \pm 2.60$ & $2.27 \pm 0.33$ & \\
\hline
\end{tabular}


A

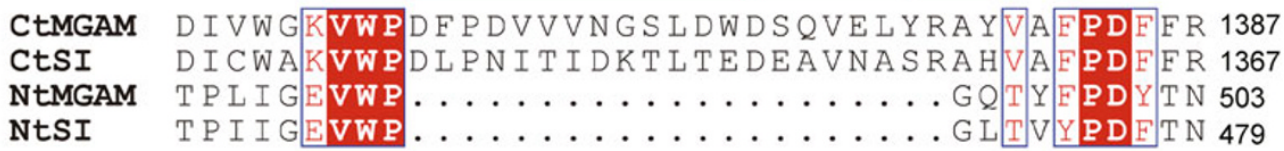

B

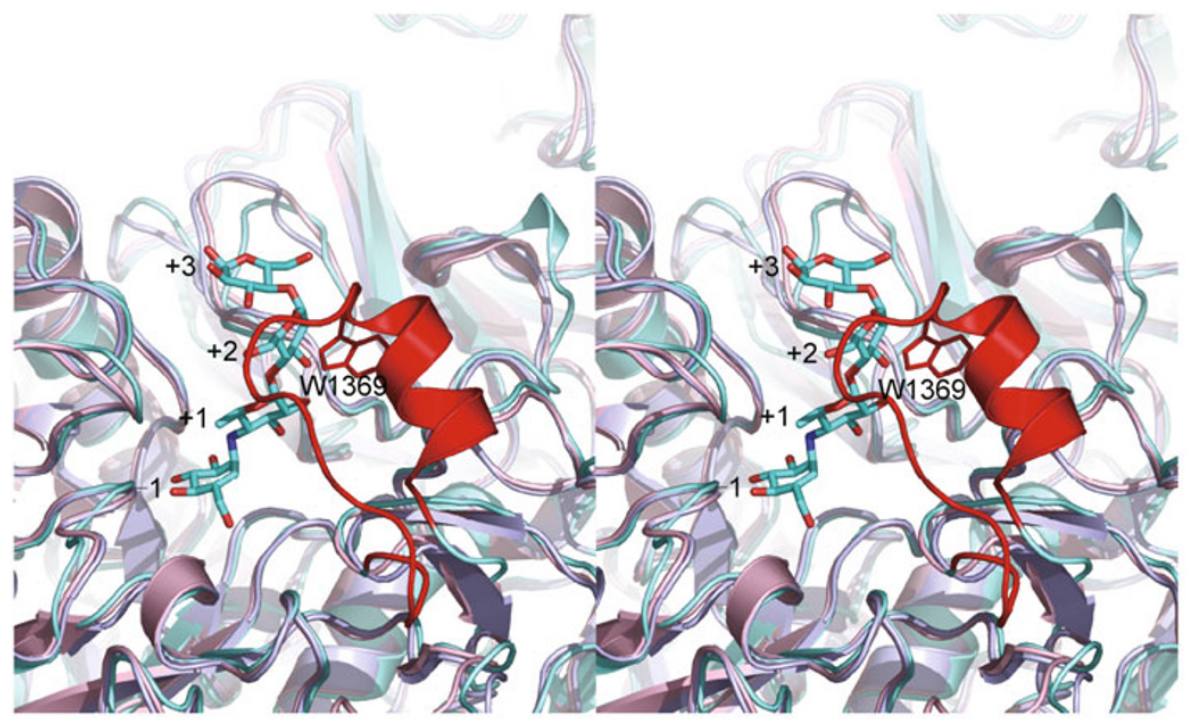

C

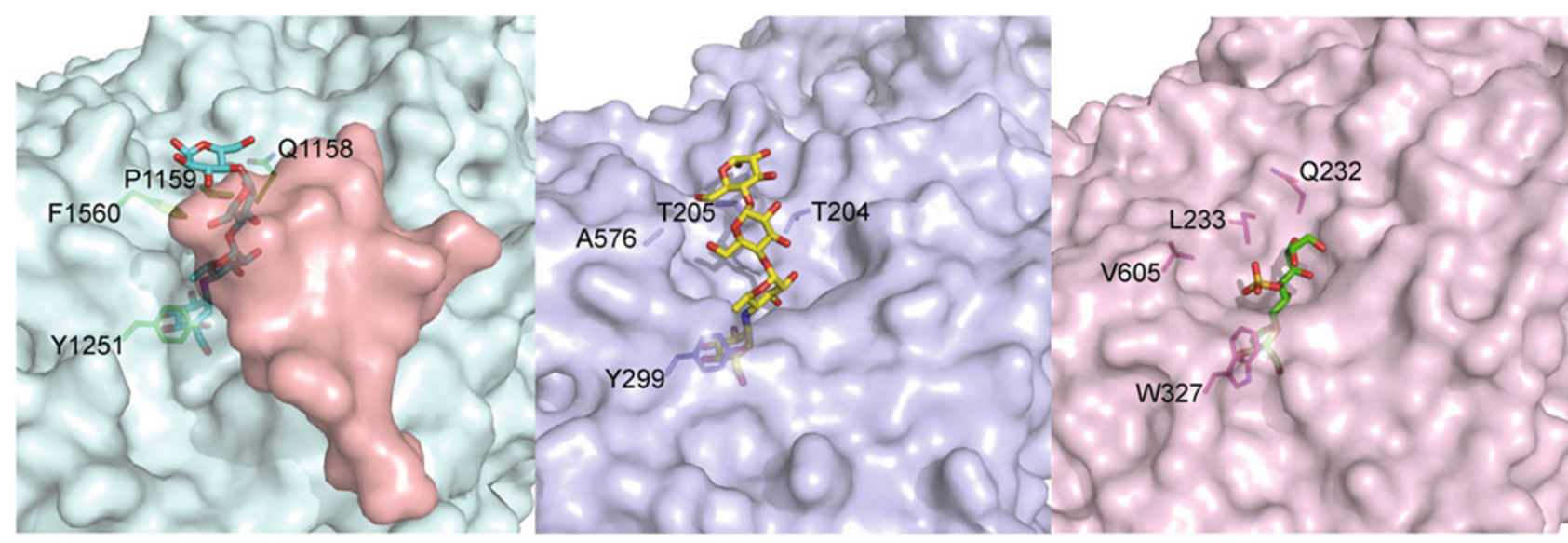

Figure 3. Substrate specificity of MGAM-C. (A) Sequence alignment of crucial insertions in MGAM and SI enzymes. Highly conserved residues are colored white, and moderately conserved residues are colored red. (B) Superposition of MGAM-C/acarbose (light cyan), MGAM-N (light purple) and SI-N (pink) active sites. Acarbose is represented as thick cyan sticks, and the additional 21 amino acids in MGAM-C are colored red. The approximate locations of the -1 to +3 subsites in MGAM-C are labeled. (C) Surface representation of the MGAM-C (light cyan)/acarbose (cyan), MGAM-N (light purple)/acarbose (yellow) and SI-N (pink)/kotalanol (green) active sites, with non-structurally conserved residues displayed as green, purple and magenta sticks, respectively. The surface of the additional 21 amino acids in MGAM-C is colored red.

To uncover the molecular mechanism underlying the substrate specificity of the MGAM enzyme, we aligned the sequences of MGAM-N, MGAM-C, SI-N and SI-C. The main difference between the sequences is a segment of 21 additional amino acids that is observed in the first insert of MGAM-C and SI-C but not in MGAM-N or SI-N (Fig. 3A).
Structural comparison of MGAM-C, MGAM-N and SI-N revealed that these extra residues folded into an $\alpha$-helix and a loop in the active site (Fig. 3B), together with other parts of MGAM-C, thereby forming +2 and +3 subsites. In our complex structure of MGAM-C/acarbose, the residue Trp1369 in the extra 21 amino acids makes a substantial 
contribution to stabilizing the third acarbose ring at the +2 subsite (Fig. 3B). These results indicate that this sequence of an extra 21 amino acids makes significant contributions to the binding of MGAM-C with longer substrates. To test this hypothesis, we made a mutant with a deletion of these extra 21 amino acids in MGAM-C (referred to as MGAM-C-deltaS) with the purpose of removing the +2 and +3 subsites of MGAM-C. As we expected, MGAM-C-deltaS showed a pattern of substrate preference similar to MGAM-N and a 5to 10 -fold reduction in binding affinities to substrates G4-G6 (Table 1).

Previous reports have shown that MGAM-C, MGAM-N, SI$\mathrm{N}$ and $\mathrm{SI}-\mathrm{C}$ have the ability to hydrolyze the linear $\alpha-1,4$-linked maltose, but only SI-N can efficiently hydrolyze the branched $\alpha-1,6$-linked isomaltose (Gray et al., 1979). To explore the functional differences between these structures at a molecular level, we compared three complex structures: MGAM-C/ acarbose, MGAM-N/acarbose, and SI-N/kotalanol. This comparison revealed approximately overlapping binding of the first ring group at the -1 subsite. Most of the amino acids at the -1 subsite are conserved, except for one residue, Tyr1251 in MGAM-C, which corresponds with Tyr299 in MGAM-N and Trp327 in SI-N (Fig. 3C). The larger hydrophobic side chain of tryptophan may reduce the space at subsite -1 and constrain the flexible branched $\alpha-1,6$-linked sugar ring to facilitate catalysis, whereas the smaller side chain of tyrosine may not efficiently stabilize the $\alpha-1,6$-linked sugar ring. To verify our hypothesis, we mutated residues Tyr1251 in MGAM-C and Tyr299 in MGAM-N to tryptophan and verified their catalytic efficiency against branched $\alpha-1,6-$ linked isomaltose. The native enzyme MGAM-C did not show catalytic activities against $\alpha-1,6$ linkages of isomaltose, but the mutant MGAM-C-Y1251W had the ability to digest an $\alpha-$ 1,6-linked substrate. The mutant enzyme MGAM-N-Y299W showed three-fold higher affinity to an $\alpha-1,6$-linked substrate than the native enzyme did. Interestingly, the two mutants MGAM-C-Y1251W and MGAM-N-Y299W had ten- and threefold reduced catalytic efficiencies against linear $\alpha-1,4$-linked substrates (Table 2). These results indicate that the mutated tyrosine residue may be involved in the swapping of catalytic activity between linear $\alpha-1,4$-linked substrates and branched $\alpha-1,6$-linked substrates.

\section{DISCUSSION}

Our study reports the crystal structure of MGAM-C. We found that the overall folding pattern of MGAM-C is similar to those of MGAM-N and SI-N, which were previously determined. As the genes for MGAM and SI are believed to have evolved by duplication of an already duplicated ancestral gene, all four subunits show a high sequence identity $(\sim 40 \%-60 \%)$ and similar digestion activities against $\alpha-1,4$-linked substrates. However, MGAM and SI have evolved differential substrate specificities and catalytic activities. MGAM-C and SI-C prefer longer substrates compared with MGAM-N and SI-N. Also, SI-N has additional activity for $\alpha-1,6$ linkages, while SI-C can digest $\alpha-1,2$ linkages. The activities and the distributions of these subunits seem to be consistent with their roles in starch digestion. The distribution ratio of $\alpha-1,4$ linkages to $\alpha-1,6$ linkages in human dietary starch molecules is 19:1. Therefore, it is necessary to have redundant $\alpha-1,4$ activities in these four enzymes, while only SI-N has high activity for $\alpha-1,6$ linkages. Sucrose, with its $\alpha-1,2$ linkages, is another important source of glucose ingestion, and in the human lumen, the ratio of SI to MGAM is almost 20 to one (Quezada-Calvillo et al., 2007). These enzymes with overlapping substrate specificities and different distributional redundancies have the combined effect of ensuring efficient starch metabolism.

A structural comparison of MGAM-C/acarbose, MGAM-N/ acarbose and SI-N/kotalanol provided us with a better understanding of the molecular mechanisms for their substrate specificities. At first, we identified +2 and +3 subsites in the catalytic center of MGAM-C but none in either MGAM-N or SI-N. The deletion of these +2 and +3 subsites in MGAM$\mathrm{C}$ altered the specificity of the enzyme, giving it similar substrate specificities to MGAM-N. This result indicated that the additional subsites in MGAM-C account for its preference for longer substrates. A sequence alignment of MGAM-C and SI-C showed $60 \%$ identity, including strong conservation of the -1 and +1 subsites. An additional 21 amino acids were also observed in SI-C; these amino acids may form +2 and +3 subsites. Additional subsites, together with other parts of the SI-C enzyme, might explain its preference for longer substrates, as with MGAM-C. Unfortunately, there is currently no crystal structure of SI-C that can be used to

Table 2 Kinetic parameters for $\alpha-1,4$ and $\alpha-1,6$ substrate hydrolysis by MGAM mutants

\begin{tabular}{|c|c|c|c|c|c|c|}
\hline \multirow[b]{2}{*}{ Substrate } & \multicolumn{3}{|c|}{ Maltose $(\alpha-1,4)$} & \multicolumn{3}{|c|}{ Isomaltose $(\alpha-1,6)$} \\
\hline & $K_{\mathrm{m}}\left(\mathrm{mmol} \cdot \mathrm{L}^{-1}\right)$ & $K_{\text {cat }}\left(\mathrm{S}^{-1}\right)$ & $\begin{array}{c}K_{\text {cat }} / K_{\mathrm{m}} \\
\left(\mathrm{S}^{-1} / \mathrm{mmol} \cdot \mathrm{L}^{-1}\right)\end{array}$ & $K_{\mathrm{m}}\left(\mathrm{mmol} \cdot \mathrm{L}^{-1}\right)$ & $K_{\text {cat }}\left(\mathrm{S}^{-1}\right)$ & $\begin{array}{c}K_{\text {cat }} / K_{\mathrm{m}} \\
\left(\mathrm{S}^{-1} / \mathrm{mmol} \cdot \mathrm{L}^{-1}\right)\end{array}$ \\
\hline MGAM-N & $6.40 \pm 0.58$ & $48.62 \pm 8.93$ & 7.57 & $70.45 \pm 6.70$ & $6.92 \pm 0.72$ & 0.10 \\
\hline MGAM-N-Y299W & $8.81 \pm 0.46$ & $23.27 \pm 0.80$ & 2.64 & $23.46 \pm 4.45$ & $4.60 \pm 0.21$ & 0.20 \\
\hline MGAM-C & $5.67 \pm 0.26$ & $22.49 \pm 0.96$ & 3.97 & N.D. & N.D. & N.D. \\
\hline MGAM-C-Y1251W & $16.16 \pm 1.49$ & $7.12 \pm 0.75$ & 0.44 & $49.95 \pm 1.03$ & $0.39 \pm 0.02$ & 0.01 \\
\hline
\end{tabular}

N.D., None detectable. 
analyze its specificity for the digestion of sucrose molecules linked by $\alpha-1,2$ bonds.

Our results showed that Trp327 at the -1 subsite of SI-N may be important in conferring $\alpha-1,6$ specificity. Mutations of Tyr to Trp in MGAM-C imparted the catalytic ability to digest branched $\alpha-1,6$-linked isomaltose. Mutating Tyr to Trp in MGAM-N also increased its affinity to $\alpha-1,6$-linked substrates compared with that of the native enzyme. The larger hydrophobic side chain of Trp stabilizes the first sugar ring as Tyr does, and further stabilizes the +1 subsite. The rigid structure of the indolyl ring of Trp constrains the flexible $\alpha-1,6$ linked sugar ring to facilitate catalysis.

Previous studies showed that many brush-border enzymes dimerize and play a part in intracellular trafficking (Danielsen, 1994). Full-length SI has been observed to homodimerize in sedimentation and electron microscopy studies (Cowell et al., 1986). Due to the large size of the molecules of this protein family, all of the solved crystal structural studies focused on individual active subunits, and there is no obvious evidence that dimers form from these subunits. Although four molecules were observed in one asymmetrical unit of the crystal structure of SI-N, the active enzyme state in the solution was detected to be a monomer. MGAM-C is notably similar to SI-N; two molecules are found in one asymmetric unit, but further analysis revealed that SI-N exists as a monomer in solution. Kinetic analysis showed that using maltose as a substrate, MGAM-N and MGAM-C have $K_{m}$ values of $6.4 \mathrm{mmol} / \mathrm{L}$ and $5.67 \mathrm{mmol} / \mathrm{L}$ respectively, while fulllength MGAM has a $K_{\mathrm{m}}$ value of $2.1 \mathrm{mmol} / \mathrm{L}$. In addition, the $K_{\mathrm{m}}$ value for mixed proteins (MGAM-N and MGAM-C) was approximately $3.65 \mathrm{mmol} / \mathrm{L}$. No significant changes were observed between independent subunits and the combined enzyme. These results suggest that MGAM-N and MGAM-C may carry out independent catalytic activities.

Amylase, MGAM and SI have been selected as drug targets for type 2 diabetes and obesity. Our results will hopefully provide important information for the design of highly efficient inhibitors of these diseases, thereby improving the health of human beings.

\section{MATERIALS AND METHODS}

\section{Cloning and expression}

The amino-acid sequences of MGAM-N and MGAM-C include residues from positions 87 to 954 and 960 to 1853 of the full-length human MGAM (Genbank Accession NM_004668.1), respectively. Their cDNA sequences were PCR amplified from a pReceiver-Y01 vector containing the gene for full-length human MGAM (from GeneCopoeia). The PCR products of MGAM-N and MGAM-C were cloned into pPIC9k expression vectors with one Avr2 restriction site. The recombinant pPic9k-MGAM-N and pPic9k-MGAM-C vectors were linearized by the restriction sites $S a / 1$ and Sac1, respectively, and were transformed into Pichia pastoris (GS115) using electroporation. The yeast strains harboring the target protein gene were screened by $\mathrm{G} 418$ selection, and the best-expression clone was found to be resistant to $\mathrm{G} 418$ concentrations of up to $4.0 \mathrm{mg} / \mathrm{mL}$. MGAM-N and MGAM-C proteins were secreted into BMMY medium when induced with methanol (up to $1 \%$ ) once every $12 \mathrm{~h}$ for a total of $72 \mathrm{~h}$. Generation of the MGAM-C-Y1251W, MGAM-C-deltaS and MGAM-N-Y299W mutants was accomplished using Dpnl-mediated site-directed mutagenesis methods in the pPic9k vector and was confirmed by DNA sequencing. The best-expression mutants were obtained following the same procedures as were used for the native enzymes.

\section{Protein purification}

The MGAM-C proteins were purified from BMMY medium by $\mathrm{Ni}$ Sepharose $^{\mathrm{TM}} 6$ Fast Flow resin (GE healthcare) $(15 \mathrm{~mL}$ resin/L media) at $4^{\circ} \mathrm{C}$. The supernatant-resin mixture was poured into a column and washed with 20 column volumes of wash buffer $(20 \mathrm{mmol} / \mathrm{L}$ Tris- $\mathrm{HCl} \mathrm{pH} 7.0$ and $200 \mathrm{mmol} / \mathrm{L} \mathrm{NaCl})$ and was eluted with elution buffer $(20 \mathrm{mmol} / \mathrm{L}$ Tris- $\mathrm{HCl} \mathrm{pH} \mathrm{7.0,200} \mathrm{mmol} / \mathrm{L} \mathrm{NaCl}$ and $300 \mathrm{mmol} / \mathrm{L}$ imidazole). The purified MGAM-C proteins were more than $95 \%$ pure, as judged by SDS-PAGE. The proteins were subsequently concentrated by Amicon filters and deglycosylated using endoglycosidase $\mathrm{F}$ overnight at room temperature in $20 \mathrm{mmol} / \mathrm{L}$ Tris- $\mathrm{HCl} \mathrm{pH} 7.0$ buffer. The presence of deglycosylated MGAM-C proteins was confirmed by SDS-PAGE. After deglycosylation, proteins were loaded onto a HiTrap Q HP anion exchange column (GE Healthcare) that was pre-equilibrated with start buffer $(20 \mathrm{mmol} / \mathrm{L}$ Tris- $\mathrm{HCl} \mathrm{pH} \mathrm{7.0)} \mathrm{and} \mathrm{eluted} \mathrm{over} \mathrm{a} \mathrm{linear} \mathrm{gradient} \mathrm{of} 0-1 \mathrm{~mol} / \mathrm{L} \mathrm{NaCl}$. The eluted proteins were concentrated to $2 \mathrm{~mL}$ and loaded onto a Superdex S200 size-exclusion column (GE Healthcare) with running buffer $(20 \mathrm{mmol} / \mathrm{L}$ Tris- $\mathrm{HCl} \mathrm{pH} 7.0,200 \mathrm{mmol} / \mathrm{L} \mathrm{NaCl})$. The total yield of pure MGAM-C was approximately $2-3 \mathrm{mg} / \mathrm{L}$ media. The protein MGAM-N and mutants were purified, as described above.

\section{Crystallization and data collection}

Crystals of MGAM-C in apo form were grown at $20^{\circ} \mathrm{C}$ using the sitting drop vapor diffusion method. Equilibrated drops were composed of $1 \mu \mathrm{L}$ protein $(5 \mathrm{mg} / \mathrm{mL}$ in $20 \mathrm{mmol} / \mathrm{L}$ Tris- $\mathrm{HCl}, \mathrm{pH} 7.0,200 \mathrm{mmol} / \mathrm{L}$ $\mathrm{NaCl})$ and $1 \mu \mathrm{L}$ reservoir buffer ( $0.3 \mathrm{~mol} / \mathrm{L} \mathrm{MgSO}_{4}, 16 \%$ PEG 3350). Diffraction-quality crystals appeared within one month and were cryoprotected with mother liquor solution supplemented with $20 \%$ glycerol. Crystals of MGAM-C in complex with acarbose were obtained by mixing acarbose $(100 \mathrm{mmol} / \mathrm{L})$ with protein $(5 \mathrm{mg} / \mathrm{mL})$ at a 1:100 volume ratio before adding the reservoir buffer as mentioned above. Diffraction data for MGAM-C crystals were collected on beam station BL17U1 at the Shanghai Synchrotron Radiation Facility (SSRF). The data were processed using the HKL2000 software package (Otwinowski and Minor, 1997).

\section{Structure determination and refinement}

The initial phase of structure determination was obtained by molecular replacement using the structure of MGAM-N (PDB code: $2 \mathrm{QLY}$ ) as a template. The program PHASER was able to locate two molecules in the asymmetric unit (McCoy, 2007). A model was built manually using the program COOT (Emsley and Cowtan, 2004) and was refined using the programs CNS (Brünger et al., 1998) and PHENIX (Adams et al., 2010). Non-crystallographic symmetry (NCS) was applied during the structure refinement procedure. Detailed data 
Table 3 Data collection and refinement statistics

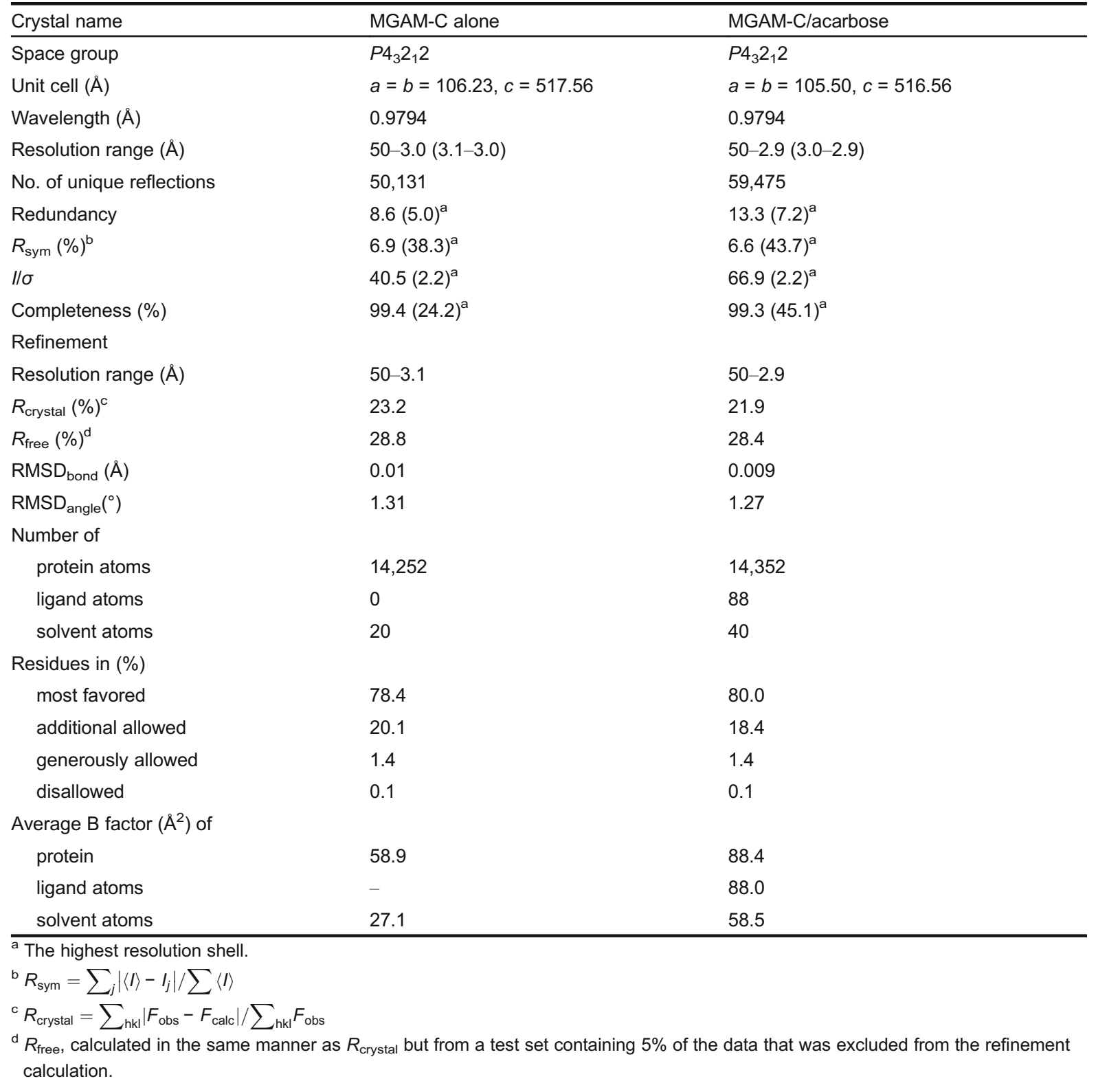

collection and refinement statistics are summarized in Table 3.

\section{Enzymatic assay}

$K_{\mathrm{m}}$ values of MGAM-N, MGAM-C, MGAM-C-deltaS for different substrates (maltose, maltotriose, maltotetraose, maltopentaose, maltohexaose, maltoheptaose and isomaltose) and MGAM-NY299W and MGAM-C-Y1251W for different substrates (maltose and isomaltose) were determined using a glucose kit (Glu Kit, Biosino Bio-Technology and Science Inc.), and assays were carried out in 96well plates. The total volume of the reaction mixture was $20 \mu \mathrm{L}$, consisting of $10 \mu \mathrm{L}$ of enzyme and $10 \mu \mathrm{L}$ of substrate $(1-80 \mathrm{mmol} / \mathrm{L}$ for MGAM-N; 0.25-40 mmol/L for MGAM-C, MGAM-C-deltaS, MGAM-N-Y299W and MGAM-C-Y1251W). As the optimal activities for MGAM-N and MGAM-C in our system occur at $\mathrm{pH} 4.8$ and $\mathrm{pH} 7.0$, respectively, all substrates and proteins used in the reaction were diluted in a buffer of either $100 \mathrm{mmol} / \mathrm{L}$ sodium acetate trihydrate $\mathrm{pH}$ 4.8 for MGAM-N and MGAM-N-Y299W, or $20 \mathrm{mmol} / \mathrm{L}$ Tris-HCl pH7.0 for MGAM-C, MGAM-C-deltaS and MGAM-C-Y1251W. The enzymatic reactions were started by the addition of MGAM-N $(0.2 \mu \mathrm{mol} / \mathrm{L})$, MGAM-C-deltaS or MGAM-C $(0.2 \mu \mathrm{mol} / \mathrm{L})$, for substrates (G2-G7). The mixture was incubated at $37^{\circ} \mathrm{C}$ for $30 \mathrm{~min}$. When using isomaltose as a substrate, the concentrations of enzyme were adjusted to $0.2-2.5 \mu \mathrm{mol} / \mathrm{L}$, and the reaction time was changed to $60 \mathrm{~min}$. Reactions were terminated by the addition of $20 \mu \mathrm{L} 2 \mathrm{~mol} / \mathrm{L}$ Tris- $\mathrm{HCl}, \mathrm{pH}$ 7.0. Next, $150 \mu \mathrm{L}$ of Glu kit reagent was added to each well at $37^{\circ} \mathrm{C}$ for $15 \mathrm{~min}$ to determinate the amount of glucose produced in the reaction. Absorbance was measured at $490 \mathrm{~nm}$. The amount of glucose production was obtained by comparing results to a standard glucose curve. $K_{m}$ values were determined by a Lineweaver-Burk plot. 
Inhibition of MGAM-C by acarbose was carried out by measuring the amount of glucose production using maltose as a substrate. The total volume of the reaction mixture was $20 \mu \mathrm{L}$, consisting of $10 \mu \mathrm{L}$ of maltose $(1-10 \mathrm{mmol} / \mathrm{L})$, differing amounts of acarbose $(0.5-2 \mu \mathrm{mol} / \mathrm{L})$ and $10 \mu \mathrm{L}$ MGAM-C. The enzymatic reactions were carried out following the above procedure. The $K i$ value for acarbose was determined by a Dixon plot.

\section{Protein data bank accession codes}

The atomic coordinates and structure factors for the structures of human MGAM-C alone and in complex with acarbose have been deposited in the PDB with accession codes 3TON and 3TOP, respectively.

\section{ACKNOWLEDGEMENTS}

This work was funded by the National Basic Research Program of China (973 Program) (Grant Nos. 2007CB914301 and 2007CB 914803), the Natural Science Foundation of China (Grant Nos. 30940015, 30770428, 21002052 and 31170684) and the TBR Program (No. 08QTPTJC 28200, 08SYSYTC00200 and 10JCYB JC14300). We are grateful to the staff at Beamline BL17U1 of Shanghai Synchrotron Radiation Facility (SSRF) for their excellent technical assistance during data collection.

Supplementary material is available in the online version of this article at http://dx.doi.org/ 10.1007/s13238-011-1105-3 and is accessible for authorized users.

\section{REFERENCES}

Adams, P.D., Afonine, P.V., Bunkóczi, G., Chen, V.B., Davis, I.W., Echols, N., Headd, J.J., Hung, L.W., Kapral, G.J., GrosseKunstleve, R.W., et al. (2010). PHENIX: a comprehensive Python-based system for macromolecular structure solution. Acta Crystallogr D Biol Crystallogr 66, 213-221.

Brayer, G.D., Luo, Y., and Withers, S.G. (1995). The structure of human pancreatic alpha-amylase at $1.8 \mathrm{~A}$ resolution and comparisons with related enzymes. Protein Sci 4, 1730-1742.

Brayer, G.D., Sidhu, G., Maurus, R., Rydberg, E.H., Braun, C., Wang, Y., Nguyen, N.T., Overall, C.M., and Withers, S.G. (2000). Subsite mapping of the human pancreatic alpha-amylase active site through structural, kinetic, and mutagenesis techniques. Biochemistry 39, 4778-4791.

Brünger, A.T., Adams, P.D., Clore, G.M., DeLano, W.L., Gros, P., Grosse-Kunstleve, R.W., Jiang, J.S., Kuszewski, J., Nilges, M., Pannu, N.S., et al. (1998). Crystallography \& NMR system: A new software suite for macromolecular structure determination. Acta Crystallogr D Biol Crystallogr 54, 905-921.

Cowell, G.M., Tranum-Jensen, J., Sjöström, H., and Norén, O. (1986). Topology and quaternary structure of pro-sucrase/isomaltase and final-form sucrase/isomaltase. Biochem J 237, 455- 461.

Dahlqvist, A., and Telenius, U. (1969). Column chromatography of human small-intestinal maltase, isomaltase and invertase activities. Biochem J 111, 139-146.

Danielsen, E.M. (1994). Dimeric assembly of enterocyte brush border enzymes. Biochemistry 33, 1599-1605.

Emsley, P., and Cowtan, K. (2004). Coot: model-building tools for molecular graphics. Acta Crystallogr D Biol Crystallogr 60, 2126-2132.

Ernst, H.A., Lo Leggio, L., Willemoës, M., Leonard, G., Blum, P., and Larsen, S. (2006). Structure of the Sulfolobus solfataricus alphaglucosidase: implications for domain conservation and substrate recognition in GH31. J Mol Biol 358, 1106-1124.

Gray, G.M., Lally, B.C., and Conklin, K.A. (1979). Action of intestinal sucrase-isomaltase and its free monomers on an alpha-limit dextrin. J Biol Chem 254, 6038-6043.

Heymann, H., Breitmeier, D., and Günther, S. (1995). Human small intestinal sucrase-isomaltase: different binding patterns for maltoand isomaltooligosaccharides. Biol Chem Hoppe Seyler 376 , 249-253.

Jenkins, D.J., Taylor, R.H., Goff, D.V., Fielden, H., Misiewicz, J.J., Sarson, D.L., Bloom, S.R., and Alberti, K.G. (1981). Scope and specificity of acarbose in slowing carbohydrate absorption in man. Diabetes 30, 951-954.

Lee, B., and Richards, F.M. (1971). The interpretation of protein structures: estimation of static accessibility. J Mol Biol 55, 379-400.

Low, L.C. (2010). The epidemic of type 2 diabetes mellitus in the AsiaPacific region. Pediatr Diabetes 11, 212-215.

McCoy, A.J. (2007). Solving structures of protein complexes by molecular replacement with Phaser. Acta Crystallogr D Biol Crystallogr 63, 32-41.

Nichols, B.L., Avery, S., Sen, P., Swallow, D.M., Hahn, D., and Sterchi, E. (2003). The maltase-glucoamylase gene: common ancestry to sucrase-isomaltase with complementary starch digestion activities. Proc Natl Acad Sci U S A 100, 1432-1437.

Nichols, B.L., Eldering, J., Avery, S., Hahn, D., Quaroni, A., and Sterchi, E. (1998). Human small intestinal maltase-glucoamylase cDNA cloning. Homology to sucrase-isomaltase. J Biol Chem 273, 3076-3081.

Nichols, B.L., Quezada-Calvillo, R., Robayo-Torres, C.C., Ao, Z., Hamaker, B.R., Butte, N.F., Marini, J., Jahoor, F., and Sterchi, E.E. (2009). Mucosal maltase-glucoamylase plays a crucial role in starch digestion and prandial glucose homeostasis of mice. J Nutr 139, 684-690.

Otwinowski, Z., and Minor, W. (1997). Processing of X-ray Diffraction Data Collected in Oscillation Mode. Methods Enzymol 276, 307-326.

Qin, X., Ren, L., Yang, X., Bai, F., Wang, L., Geng, P., Bai, G., and Shen, Y. (2011). Structures of human pancreatic a-amylase in complex with acarviostatins: Implications for drug design against type II diabetes. J Struct Biol 174, 196-202.

Quezada-Calvillo, R., Robayo-Torres, C.C., Opekun, A.R., Sen, P., Ao, Z., Hamaker, B.R., Quaroni, A., Brayer, G.D., Wattler, S., Nehls, M.C., et al. (2007). Contribution of mucosal maltaseglucoamylase activities to mouse small intestinal starch alphaglucogenesis. J Nutr 137, 1725-1733.

Quezada-Calvillo, R., Sim, L., Ao, Z., Hamaker, B.R., Quaroni, A., Brayer, G.D., Sterchi, E.E., Robayo-Torres, C.C., Rose, D.R., and Nichols, B.L. (2008). Luminal starch substrate "brake" on maltaseglucoamylase activity is located within the glucoamylase subunit. J Nutr 138, 685-692.

Rabasa-Lhoret, R., and Chiasson, J.L. (1998). Potential of alphaglucosidase inhibitors in elderly patients with diabetes mellitus and impaired glucose tolerance. Drugs Aging 13, 131-143.

Rossi, E.J., Sim, L., Kuntz, D.A., Hahn, D., Johnston, B.D., Ghavami, 
A., Szczepina, M.G., Kumar, N.S., Sterchi, E.E., Nichols, B.L., et al. (2006). Inhibition of recombinant human maltase glucoamylase by salacinol and derivatives. FEBS J 273, 2673-2683.

Semenza, G. (1986). Anchoring and biosynthesis of stalked brush border membrane proteins: glycosidases and peptidases of enterocytes and renal tubuli. Annu Rev Cell Biol 2, 255-313.

Sim, L., Quezada-Calvillo, R., Sterchi, E.E., Nichols, B.L., and Rose, D.R. (2008). Human intestinal maltase-glucoamylase: crystal structure of the $\mathrm{N}$-terminal catalytic subunit and basis of inhibition and substrate specificity. J Mol Biol 375, 782-792.

Sim, L., Willemsma, C., Mohan, S., Naim, H.Y., Pinto, B.M., and Rose, D.R. (2010). Structural basis for substrate selectivity in human maltase-glucoamylase and sucrase-isomaltase $\mathrm{N}$-terminal domains. J Biol Chem 285, 17763-17770.

Van Beers, E.H., Büller, H.A., Grand, R.J., Einerhand, A.W., and Dekker, J. (1995). Intestinal brush border glycohydrolases: structure, function, and development. Crit Rev Biochem Mol Biol 30, 197-262. 